\title{
An Anthropometric Comparison of Different Player Positions in Elite Rugby Union Sevens
}

\author{
Pieter H. VAN DEN BERG ${ }^{1}$, J. Hans DE RIDDER ${ }^{1 *}$, Dawie D.J. MALAN ${ }^{1}$, Suria M ELLIS ${ }^{2}$ \\ ${ }^{I}$ Physical Activity, Sport and Recreation Focus Area (PhASRec), Faculty of Health Sciences, North-West \\ University, Potchefstroom, 2520, South Africa. \\ ${ }^{2}$ Unit for Business Mathematics and Informatics, Faculty of Health Sciences, North-West University, \\ Potchefstroom, 2520, South Africa.
}

*Corresponding Author: J. Hans de Ridder, Ph.D. Director and Professor, School of Human Movement Sciences, North-West University, Potchefstroom Campus, Potchefstroom, 2520 SOUTH AFRICA.

\begin{abstract}
:
Background: An anthropometric profile acts as an important morphological characteristic that affects the performance of each rugby player. Players in different positions have diverse responsibilities, consequently leading to different anthropometric profiles. The purpose of this study was to compare the different player position subgroups of elite, national, Rugby Union Sevens (RS) players with regard to their anthropometric profiles.
\end{abstract}

Methods: Positional subgroups were divided into forward and backline players. The total group $(n=15)$ of the national elite RS players of Zimbabwe participated in the study. Stature, body mass, skinfolds, girths and breadths were measured through the standard procedures as described by ISAK. Different body composition parameters as well as the different somatotypes were calculated.

Results: Significant differences were found between the forward and backline players with regard to stature, body mass, forearm girth, femur breadth and mesomorphy. In addition, backline players had significantly less muscle mass $(\mathrm{kg})$ and \% muscle mass when compared to the forward players. The forward players in the current study had a practical significantly higher mesomorphy value, compared to the backline players.

Conclusion: The study highlighted that RS requires players with different body types and anthropometric characteristics to adhere to the responsibilities set for the different player positional subgroups.

Keywords: Sevens Rugby, anthropometry, somatotypes, body composition, Rugby Union.

Running title: Anthropometry of elite rugby sevens

\section{INTRODUCTION}

Rugby Union Sevens (RS) host their own respective World Cup every four years [1]. The sport recently received Olympic status to be included as an event from 2016 in Rio de Janeiro, Brazil Olympics [2]. Achieving Olympic status have motivated international teams to pursue performance even more in the quest to win an Olympic medal [3]. Research suggests therefore, that in order to improve performance, it is important to understand the physiological and morphologial demands placed on the players [4,5]. Sport scientists [6] suggested that the anthropometric profiles of rugby players should be investigated, since morphological characteristic may affect the performance of these players. It has been proven that such anthropometric evaluation has a direct bearing on the selection processes and performance of rugby players $[7,3,8,9]$.

When anthropometric measurements are conducted, it is important to bear in mind that different players are allocated to different player positions. Each position has its own specific functions and responsibilities during a match, which can be related to the anthropometric profile of these players $[10,11,12]$. Literature on Rugby Union suggests that when position-specific differences are investigated, the players should be subdivided into forward and backline players [10,11]. Hence the following research question: How does the two different player positional subgroups of the Zimbabwean National RS players, compare to one another with regard to their anthropometric profiles? 
The results of the current study could enable coaches, sport scientists, officials, selectors and players to better understand the possible influence position-specific anthropometric profiles of players in a senior national RS team, may have on their playing performance. The knowledge gathered from this study may therefore indirectly lead to position-specific physical assessment and training programs of the players[13] and also offer valuable assistance to coaches with team selection [14,15].

\section{METHODS}

\subsection{Participants and Ethics}

All the senior male players of the Zimbabwean national RS team $(n=15)$ with a mean age of 27.2 years were tested. Players were divided into forward $(n=6)$ and backline players $(n=9)$. Before commencement of the project, all the players completed an informed consent form once the study protocol had been explained to them. The management of the team also had to grant their approval for the project. It was explained to the players that participation was done on a voluntary basis and any player could withdraw at any time if they so wished. The study received ethical approval from the Ethics Committee in the Faculty of Health Sciences of the North-West University [NWU-00122-11A1].

\subsection{Procedures and Measurements}

Seventeen anthropometric measurements were taken on the players of the Zimbabwean National RS team, by two ISAK internationally accredited level II anthropometrists, assisted by data recorders. All measurements (stature, body mass, girths, skinfolds and breadths) were taken in accordance with the standard procedures as described by the International Standards for Anthropometric Assessment [16]. All the data were collected in the mornings prior to any physical activity.

Body mass was measured with a calibrated electronic scale (Precision, A\&D Company, Saitama, Japan) to the nearest $0.1 \mathrm{~kg}$ [16]. Stature was measured with a stadiometer to the nearest $0.1 \mathrm{~cm} \mathrm{[16].}$ Six skinfold sites (triceps, subscapular, supraspinale, abdominal, thigh and calf) were measured to the nearest $0.2 \mathrm{~mm}$ by using a Harpenden skinfold caliper [16]. Breadths $(\mathrm{cm})$ (femur, humerus, wrist and ankle) were measured with Cescorf bone calipers to the nearest $0.1 \mathrm{~cm}$. Girths (forearm, mid-thigh and calf) were measured by using a flexible steel tape (Cescorf) to the nearest $0.1 \mathrm{~cm}$. All measurements were taken twice, with a third measurement when any of the 2 measurements were outside the allowed limits. In the case of two measurements, the mean was taken as the official reading whereas the median was used in the case of three measurements [16].

\section{Body composition:}

Body mass and stature were used to calculate the Body Mass Index (BMI) [17].

- $\quad$ BMI = MASS $(\mathbf{k g}) / \operatorname{STAT}^{2}(\mathbf{m})$.

The fat percentage was determined by means of the equation of Whithers et al. (1987) [18].

- $\quad$ Body density $(\mathrm{BD})=1.10326-0.00031($ Age $)-0.00036\left(\sum 6\right.$ skinfolds $)$.

- $\% \mathrm{BF}=(495 / \mathrm{BD})-450$

- $\quad \sum 6$ skinfolds $=($ triceps + subscapular + supraspinale + abdominal + front thigh + medial calf)

Skeletal mass ( $\mathrm{kg}$ ) was calculated according to the equation of Martin (1991) [19].

- $\quad$ [0.00006 x STAT x (femur breadth + humerus breadth + wrist breadth + ankle breadth) $\left.{ }^{2}\right]$.

Lee et al. (2000) [20] suggested that muscle mass $(\mathrm{kg})$ and percentage muscle mass needed to be calculated as follows.

- STAT (0.00744 $\times$ CAG $^{2}+0.00088 \times$ CTG $^{2}+0.0044 \times$ CCG $\left.^{2}\right)+2.4 \times($ gender $)-0.048 \times($ Age $)$ + race +7.8

xWhere:

- $\mathrm{CAG}=$ corrected arm girth (relaxed);

- $\mathrm{CTG}=$ corrected thigh girth (mid-thigh);

International Journal of Sports and Physical Education (IJSPE) 
- $\mathrm{CCG}=$ corrected calf girth;

- $\quad$ STAT = stature;

- $\quad$ MASS = Body mass;

Race $=-2$ for Asians, 1.1 for African American and 0 for White and Hispanic;

Corrected muscle circumferences $=\mathrm{C}_{\mathrm{m}} ; \mathrm{C}_{\mathrm{m}}=\mathrm{Climb}-3.1416 \mathrm{x}($ skinfold $/ 10)$.

\section{Somatotyping:}

The different somatotypes were calculated to the nearest 0.1 by using the equations as suggested by Carter and Heath (1990) [21]. The somatotypes for the individuals and for the mean total of all the players in each positional subgroup were calculated as follows:

- Endomorphy $=\mathbf{- 0 . 7 1 8 2}+\mathbf{0 . 1 4 5 1}\left(\sum\right.$ of skinfolds $x$ 170.18/Stature $)-0.00068\left(\sum\right.$ of skinfolds $x$ 170.18/stature $)^{2}+\mathbf{0 . 0 0 0 0 0 1 4}\left(\sum \text { of skinfolds x 170.18/stature }\right)^{3}$

Where: $\sum$ of skinfolds $=$ triceps, subscapular and supraspinale skinfolds.

- Mesomorphy $=0.858($ HUMB $)+0.601($ FEMB $)+($ CAG $)+0.161($ CCG $)-0.131($ STAT $)+4.5$

Where: $\quad$ HUMB $=$ humerus breadth $(\mathrm{cm})$;

FEMB = femur breadth $(\mathrm{cm})$;

$\mathrm{CAG}=$ corrected arm girth (flexed arm girth $[\mathrm{cm}]-$ triceps skinfold $[\mathrm{mm}] / 10$ );

$\mathrm{CCG}=$ corrected calf girth $($ calf girth $[\mathrm{cm}]-$ calf skinfold $[\mathrm{mm}] / 10)$;

STAT $=$ stature

- $\quad$ Ectomorphy $=($ HWR $\times 0.732)-28.58$

Where: $\quad($ HWR $)=$ STAT $/ 3 \sqrt{ }$ MASS;

Note: if HWR $<40.75$, but $>38.25$ then Ectomorphy $=$ HWR $\times 0.463-17.63$ and if HWR $\leq 38.25$ then Ectomorphy $=0.1$. A three-number somatotype rating was plotted on a two-dimensional somatochart using $\mathrm{X}$ and $\mathrm{Y}$ coordinates.

The coordinates were calculated as follows:

- $\quad X=$ ectomorphy - endomorphy

- $\quad \mathrm{Y}=2 \mathrm{x}$ mesomorphy - (endomorphy + ectomorphy) [21].

\subsection{Statistical Analysis}

The statistical software packages from SPSS Inc. (2013) [22] and Statsoft Inc. (2013) [23] were used to analyse the data. Descriptive statistics were initially determined to give an indication of anthropometric profiles of all the players. Due to the small population size, non-parametric data analysis was performed with the Mann-Whitney test to compare the data between the player's positional groups.

During the exploration of the data, Q-Q plots were drawn to determine normality, by eyeballing the plots. From this, all the variables seemed to be normally distributed.As this study does not consist of a random sample, statistical significance (p-values) was not relevant and were only calculated for completeness. The interpretation of the non-parametric effect size were therefore applied to determine whether these differences were important in practice. Emphasis were placed on differences that provided a large practical significant value. Guidelines for the interpretation of $r: r \geq 0.1$ small significance; $r \geq 0$.3medium significance and $r \geq 0.5$ large significance [24].

\section{Results}

The results of the descriptive statistics of the total group senior elite Zimbabwean national RS players, as well as the data of the forwards and backs are presented in Table 1. Also presented in Table 1 are the results of the Mann-Whitney test as well as the interpretation of the non-parametric effect size. Emphasis were placed on differences that provided a large practical significant value. 
An Anthropometric Comparison of Different Player Positions in Elite Rugby Union Sevens

Table1. Comparison of anthropometric data between the player positional subgroups in the senior Zimbabwean national RS team

\begin{tabular}{|l|l|l|l|l|l|l|l|l|}
\hline & \multicolumn{9}{|l|}{$\begin{array}{l}\text { Total Group } \\
(\mathbf{n = 1 5})\end{array}$} & \multicolumn{2}{l}{$\begin{array}{l}\text { Forwards } \\
(\mathbf{n = 6})\end{array}$} & \multicolumn{2}{l|}{$\begin{array}{l}\text { Backs } \\
(\mathbf{n = 9})\end{array}$} & $\begin{array}{l}\text { Mann- } \\
\text { Whitney }\end{array}$ & $\begin{array}{l}\text { Effect } \\
\text { size }\end{array}$ \\
\hline Variables & mean & SD & mean & SD & mean & SD & p-value & r-value \\
\hline Stature (cm) & 178.44 & 4.79 & 183.01 & 6.7 & 174.31 & 4.9 & 0.67 & 0.5 \\
\hline Body mass (kg) & 84.16 & 8.31 & 88.7 & 7 & 81.13 & 8.5 & 0.67 & 0.5 \\
\hline BMI & 26.41 & 1.7 & 26.47 & 1.5 & 26.36 & 2.0 & 0.77 & 0.1 \\
\hline \% Body fat & 9.46 & 2.77 & 9.45 & 1.7 & 9.47 & 3.4 & 0.53 & 0.2 \\
\hline \% Muscle mass & 41.62 & 2.18 & 42.66 & 0.7 & 40.93 & 2.6 & 0.11 & 0.5 \\
\hline \% Skeletal mass & 11.47 & 0.80 & 11.38 & 1.1 & 11.54 & 0.6 & 0.78 & 0.1 \\
\hline $\begin{array}{l}\text { Muscle mass } \\
\text { (kg) }\end{array}$ & 37.29 & 2.15 & 38.39 & 1.3 & 36.55 & 2.4 & 0.18 & 0.6 \\
\hline $\begin{array}{l}\text { Skeletal mass } \\
\text { (kg) }\end{array}$ & 9.65 & 1.14 & 10.09 & 1.2 & 9.36 & 1 & 0.33 & 0.2 \\
\hline$\sum$ 6 skinfolds & 50.30 & 17.16 & 50.45 & 10.3 & 50.2 & 21.2 & 0.53 & 0.2 \\
\hline Triceps SF & 9.21 & 11.22 & 5.87 & 0.8 & 6.53 & 2.8 & 1.00 & 0.0 \\
\hline Subscapular SF & 10.41 & 3.80 & 9.82 & 0.3 & 10.81 & 4.8 & 0.69 & 0.1 \\
\hline Supraspinale SF & 6.55 & 2.46 & 6.82 & 1.24 & 6.38 & 3 & 0.18 & 0.4 \\
\hline Abdominal SF & 11.63 & 5.31 & 11.83 & 3.8 & 11.49 & 6.4 & 0.53 & 0.2 \\
\hline Front thigh SF & 8.77 & 3.30 & 9.38 & 3.6 & 8.36 & 3.2 & 0.69 & 0.1 \\
\hline Medial calf SF & 6.87 & 2.10 & 7.23 & 1.51 & 6.63 & 2.47 & 0.61 & 0.2 \\
\hline Arm GR flexed & 38.47 & 2.04 & 38.8 & 1.71 & 38.26 & 2.3 & 0.69 & 0.1 \\
\hline Arm GR relax & 34.24 & 3.24 & 35.17 & 1.7 & 33.62 & 3.9 & 0.33 & 0.3 \\
\hline Calf GR & 37.73 & 2.21 & 37.55 & 2.4 & 37.84 & 2.2 & 0.86 & 0.1 \\
\hline Forearm GR & 29.98 & 2.02 & 30.8 & 0.5 & 29.43 & 2.5 & 0.05 & 0.5 \\
\hline Mid-thigh GR & 58.10 & 2.39 & 58.5 & 2.6 & 57.83 & 2.4 & 0.46 & 0.2 \\
\hline Humerus BR & 7.45 & 0.45 & 7.48 & 0.5 & 7.42 & 0.4 & 0.61 & 0.2 \\
\hline Femur BR & 9.61 & 0.62 & 10.1 & 0.56 & 9.47 & 0.76 & 0.60 & 0.5 \\
\hline Wrist BR & 5.79 & 0.34 & 5.82 & 0.3 & 5.77 & 0.4 & 0.78 & 0.1 \\
\hline Ankle BR & 7.14 & 0.31 & 7.30 & 0.3 & 7.03 & 0.3 & 0.14 & 0.4 \\
\hline
\end{tabular}

$S F=$ Skinfold $(\mathrm{mm}) ; G R=$ Girth $(\mathrm{cm}) ; B R=$ Breadth $(\mathrm{cm}) ; r \geq 0.5$ practical significance;

The data in Table 1 indicated that the forwards were taller and heavier than the backs, with both these measurements showing practical significance (body mass: $r=0.5$ and stature: $r=0.5$ ). The forwards also had a larger muscle mass than the backs with practical significance both for the muscle mass in $\mathrm{kg}$ $(r=0.5)$ as well as the percentage muscle mass $(r=0.6)$. Only small practical differences were found for the BMI, the percentage body fat, the sum of 6 skinfolds as well as the skeleton mass between the forwards and the backs.

With regard to the skinfolds, the forwards had larger supraspinale, abdominal, thigh and calf skinfolds with the backs who had larger triceps and subscapular skinfolds, than the forwards. None of these differences were practically significant with only supraspinale skinfold showing a medium practical difference and the rest of the skinfolds showing a small practical differences.

The forwards had larger girths than the backs in 4 of the measurements (arm flexed, arm relaxed, forearm and mid-thigh). Although the backs had larger calf girths than the forwards, there were only a small practical difference between the two groups. The only girth showing a practical significant $(\mathrm{r}=0.5)$ difference between the forwards and the backs, was the forearm girth. As expected, the forwards showed larger breadths thank the backs in al four the measurements with a practically significant $(\mathrm{r}=0.44)$ larger femur breadth than the backs.

The comparisons of the somatotypes between the forwards and the backs (different positional subgroups) are presented in Table 2 with a plotting of the mean somatotypes for each subgroup presented in Figure 1. 
Table2. Comparison of somatotypes between the different positional subgroups of Zimbabwean national RS players $(n=15)$

\begin{tabular}{|l|l|l|l|l|l|l|l|}
\hline & \multicolumn{2}{|l}{$\begin{array}{l}\text { Forward players } \\
(\mathbf{n = 6})\end{array}$} & \multicolumn{2}{l|l}{$\begin{array}{l}\text { Backline players } \\
(\mathbf{n = 9 )}\end{array}$} & t-test & $\begin{array}{l}\text { Mann- } \\
\text { Whitney }\end{array}$ & $\begin{array}{l}\text { Effect } \\
\text { sizes }\end{array}$ \\
\hline Variable & mean & SD & mean & SD & p-values & p-value & r-value \\
\hline Endomorphy & 2.2 & 1.07 & 2.0 & 0.36 & 0.64 & 0.86 & 0.1 \\
\hline Mesomorphy & 6.7 & 0.89 & 6.0 & 0.49 & 0.07 & 0.11 & 0.5 \\
\hline Ectomorphy & 1.3 & 0.6 & 1.6 & 0.67 & 0.40 & 0.33 & 0.3 \\
\hline
\end{tabular}

$r \geq 0.5$ practical significance

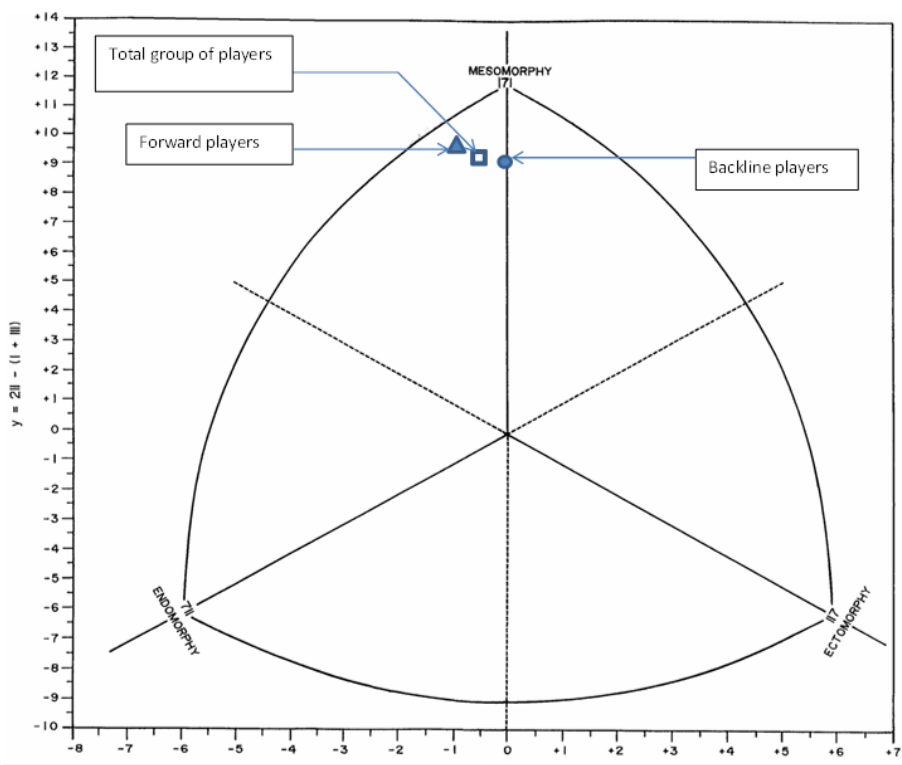

Figure1. Somatochart with somatotypes for the total and different positional subgroups of Zimbabwean national $R S$ players

The Somatotyping of players (related to body build) could provide more insight into the players' morphological profile and specifically their type of body build [21].

The forward players in the current study had a practical significantly $(r=0.49)$ higher mesomorphic value compared to that of the backline players. However, both positional subgroups tend to be dominant mesomorphic in general, with the average somatotype for the total group of players (2.16.7-1.2) as well as the forwards (2.2-6.7-1.3), classified as endomorphic mesomorphs. The backs (2.06.0-1.6) were classified as balanced mesomorphs. On the somatochart the total group of players $(n=15)$ as well as the forwards $(n=6)$ and the backs $(n=9)$, were plotted as dominant mesomorphs (see Figure 1).

\section{DISCUSSIONS}

The average height $(178.4 \mathrm{~cm})$ of the Zimbabwean RS player, is comparable to that of rugby players in similar research projects $[25,26,27,28]$, focusing on sevens rugby. The average body mass $(84.1 \mathrm{~kg})$ of the Zimbabwean RS players, is also in the proximity of the body mass values reported by similar studies on sevens players $[25,26,27,28]$. Backline players in the current study had a fat percentage of 9.46 , which is comparable to the $9.2 \%$ found amongst the national RS backline players of the United States of America [3].

Taking into consideration that the previously mentioned studies focused on elite teams from different nationalities, one can assume that elite international RS players presented an almost similar body mass and stature irrespective of nationality. The similarity in body mass and stature of the different international RS players may be accredited to the management teams to select players that may adapt to the physique of the opponents. Coaches and selectors may have developed a similar philosophy of the ideal build or morphology for RS players to enable them to perform optimally.

The practical significant differences between the stature as well as the body mass of the forwards and the backs, are similar to the findings of Rienzi et al., (1999) [29] and Fuller et al. (2010) [1]. Forward players showed higher values of body mass when compared to the backline players. The practically 
significant smaller backline players in the present study (as indicated by the smaller muscle mass and $\%$ muscle mass) are in line with results from Rienzi et al. (1999) [29], who found forward RS players to have more muscle mass than backline players. The lighter backline players in the current study may be linked to a higher degree of work output for these players, when compared to the heavier players [30]. The additional body mass as well as muscle mass of the forward players may be explained by the different responsibilities of the respective players such as scrumming and lifting other players at line-outs and at kick-offs. The backline players on the other hand, may be able to continue for longer periods on high-intensity running activities due to their lower body mass.

The practical significant difference found between the forearm girth of forward and backline players may be attributed to the necessity of the forward players to bind during scrums, line-out and kick-off support where a bigger forearm could lead to increased grip strength. The bigger femur breadth values of the forward players may be attributed to the forward players being superior in stature and mass when compared to the backline players. Bigger players require proportionally bigger femurs. No practical significant differences were found for any of the other anthropometrical measurements. As far as the somatotypes are and concerned, the forward players in the current study had a practical significant $(r=0.49)$ higher mesomorphic value than the backline players which is similar to the findings of Quarrie et al. (1996) [6], De Ridder et al. (2020) [31], as well as Holway \& Garavaglia (2009) [9]. All three these studies determined that forward players in Rugby Union presented significantly superior mesomorphic values in comparison to the backline players. However, both player subgroups in the current study are dominant mesomorphs, with the average somatotype of the whole group as endomorphic mesomorph (2.1-6.7-1.2). This finding correlates with that of De Ridder et al. (2020) [31].

No significant differences were found in the BMI of the Zimbabwean RS players amongst the different player positional subgroups. An interesting observation was that the average BMI value (26.4) for the total group of Zimbabwean RS players in the present study is very similar to the 26.1 reported by Elloumi et al. (2012) [26] for Tunisian RS players.

\section{CONCLUSION}

When the anthropometric measurements of the Zimbabwean RS players in the positional subgroups were compared, forward players were significantly heavier and presented bigger stature, forearm girth, femur breadth and mesomorphy values than backline players. In addition backline players had less muscle mass $(\mathrm{kg})$ and \%muscle mass than forward players. These anthropometric differences between the player positions could be attributed to the different responsibilities of each player's positional group.

In addition to the objective of the present study, a comparison of results between previous research and results from the current study suggests that RS players seem to be a homogenous group of players, despite their respective nationalities. The study highlighted that RS requires players with different anthropometric profiles to adhere to the responsibilities as set for the different player position subgroups.

\section{FUNDING}

The authors of this study would like to thank the South African National Lottery Distribution Trust Fund (NLDTF) that provided the financial support for the research.

\section{REFERENCES}

[1] Fuller, C.W., Taylor, A., \& Molloy, M.G. (2010). Epidemiological study of injuries in international rugby sevens. Clinical Journal of Sport Medicine, 20(3), 179-184.

[2] Engebretsen, L., \& Steffen, K. (2010). Rugby in Rio. British Journal of Sports Medicine, 44(3), 157.

[3] Carlson, B.R., Carter, J.E.L., Patterson, P., Petti, K., Orfanos, S.M., \& Noffal, G.J. (1994). Physique and motor performance characteristics of US national rugby players. Journal of Sports Sciences, 12(4), 403412.

[4] Nicholas, C.W. (1997). Anthropometric and physiological characteristics of rugby union football players. Sports Medicine, 23(6), 375-396.

[5] Cunniffe, B., Proctor, W., Baker, J.S \& Davies, B. (2009). An evaluation of the physiciological demands of elite rugby union using global positioning system tracking software. Journal of strength and conditioning research, 23(4), 1195-1203. 
[6] Quarrie, K.L., Handcock, P., Waller, A.E., Chamers, D.J., Toomey, M.J., \& Wilson, B.D. (1995). The New Zealand rugby injury and performance project III. Anthropometric and physical performance characteristics of players. British Journal of Sports Medicine, 29(4), 263-270.

[7] Bell, W. (1979). Body composition of rugby union football players. British Journal of Sports Medicine, 13(1), 19-23.

[8] Olds, T. (2001). The evolution of physique in male rugby union players in the twentieth century. Journal of Sports Sciences, 19(4), 253-262.

[9] Holway, F.E., \& Garavaglia, R. (2009). Kinanthropometry of Group I rugby players in Buenos Aires, Argentina. Journal of Sports Sciences, 27(11), 1211-1220.

[10] Duthie, G., Pyne, D., \& Hooper, S. (2003). Applied physiology and game analysis of rugby union. Sports Medicine, 33(13), 973-991.

[11] Gamble, P. (2004). Physical preparation for elite-level rugby union football. Strength and Conditioning Journal, 26(4), 10-23.

[12] Eaton, C., \& George, K. (2006). Position specific rehabilitation for rugby union players. Part1:Emperical movement analysis data. Physical Therapy in Sport, 7(1), 22-29.

[13] Coughlan, G.F., Green, B.S., Pook, P.T., Toolan, E., \& O'Connor, S.P. (2011). Physical game demands in elite rugby union: A global positioning system analysis and possible implications for rehabilitation. Journal of Orthopaedic and Sports Physical Therapy, 41(8), 600-605.

[14] Quarrie, K.L., Handcock, P., Toomey, M.J., \& Waller. D.J. (1996). The New Zealand rugby injury and performance project IV. Anthropometric and physical performance comparisons between positional categories of senior A rugby players. British Journal of Sports Medicine, 30(1), 53-60.

[15] Du Randt, J., Du Toit, S., Borresen, J., Hew-Butler, T., Masimla, H., Jakoet, I., \& Lambert, M. (2006). Fitness and body composition profiling of elite junior South African rugby players. South African Journal of Sports Medicine, 18(2), 38-45.

[16] Stewart, A., Marfell-Jones, M., Olds, T., \& De Ridder, J.H. (2011). International standards for anthropometric assessment. New Zealand: ISAK.

[17] Eston, R., Hawes, M., Martin, A., \& Reilly, T. (2009). Human body composition. In R. Eston, \& T. Reilly, (Eds), Kinanthropometry and exercise physiology laboratory manual: test, procedures and data. (pp. 3-53). London: Routledge.

[18] Whithers, R.T., Craig, N.P., Bourdon, P.C., \& Norton, K.I. (1987). Relative body fat and anthropometric prediction of body density of male athletes. European Journal of Applied Physiology, 56(2), 191-200.

[19] Martin, A.D. (1991). Anthropometric assessment of bone mineral. In L. Himes (Ed.), Anthropometric assessment of nutritional status (pp. 185-196). New York: Wiley-Liss.

[20] Lee, R.C., Wang, Z., Heo, M., Ross, R., Janssen, I., \& Heymsfield, S.B. (2000). Total body skeletal muscle mass: development and cross-validation of anthropometric prediction models. American Journal of Clinical Nutrition, 72(3), 796-803.

[21] Carter, J.E.L., \& Heath, B.H. (1990). Somatotyping: Development and applications, Cambridge: Cambridge University press.

[22] SPSS Inc. (2013). IBM SPSS Statistics Version 21, Release 21.0.0,Copyright $\odot$ IBM Corporation and its licensors. http://www-01.ibm.com/software/analytics/spss/.

[23] StatSoft, Inc. (2013). STATISTICA (data analysis software system), version 11. www.statsoft.com.

[24] Field, A. 2009. Discovering statistics using SPSS. London: Sage Publications.

[25] Walsh, J., Climstein, M., Heazlewood, I. T., Burke, S., Kettunen, J., Adams, K., \& DeBeliso, M. (2011). Improved body mass index classification for football code masters athletes: A comparison to the Australian national population. International Journal of Biological and Medical Sciences, 1(1), 37-40.

[26] Elloumi, M., Makni, E., Moalla,W., Bouaziz, T., Tabka, Z., Lac, G., \& Chamari, K. (2012). Monitoring training load and fatigue in rugby sevens players. Asian Journal of Sports Medicine, 3(3), 175-184.

[27] Suarez-Arrones, L., Arenas, C., Lopez, G., Requena, B., Terrill, O. \& Mendez-Villanueva, A. (2014). Positional differences in match running performance and physical collisions in men rugby sevens. International journal of sports physiology and performances, 3, 316-323.

[28] Higham, D.G., Pyne, D.B., Anson, J.M., \& Eddy, A. (2013). Physiological, anthropometric and performance characteristics of rugby sevens players. International Journal of Sports Physiology and Performance, 8(1),19-27.

[29] Rienzi, E., Reilly, T., \& Malkin, C. (1999). Investigation of anthropometric and work-rate profiles of Rugby Sevens players. The Journal of Sports Medicine and Physical Fitness, 39(2), 160-164.

[30] Meir, R. (2012). Training for and competing in sevens rugby: Practical considerations from experience in the International Rugby Board world series. Strenght and Conditioning Journal, 34(4), 76-86. 
[31] De Ridder, J.H., Van Den Berg, P.H., Zandberg, A., Broodryk, R. (2020).Anthropometric characteristics of elite $\mathrm{u} / 18$ Sevens rugby players: A focus on positional status. International Journal of Sports and Physical Edication (IJSPE), 6(3):16-23. http://dx.doi.org/10.20431/2454-6380.0603005

Citation: J. Hans DE RIDDER, et.al. "An Anthropometric Comparison of Different Player Positions in Elite Rugby Union Sevens" International Journal of Sports and Physical Education (IJSPE), vol 7, no. 1, 2021 , pp. 11-18. doi: https://doi.org/10.20431/2454-6380.0701003.

Copyright: (c) 2021 Authors. This is an open-access article distributed under the terms of the Creative Commons Attribution License, which permits unrestricted use, distribution, and reproduction in any medium, provided the original author and source are credited. 